\title{
Correlation of Surface Plasmon Resonance Wavelength (SPR) with Size and Concentration of Noble Metal Nanoparticles
}

\author{
Nikita Dalal' ${ }^{1}$ Bijoy Sanakr Boruah², Ashamoni Neoh ${ }^{2}$ and Rajib Biswas ${ }^{2}$ \\ ${ }^{1}$ Department of Physics, M.D. University, India \\ ${ }^{2}$ Department of Physics, Tezpur University, India
}

Submission: August 12, 2019; Published: August 29, 2019

"Corresponding author: R Biswas, Applied Optics and Photonics Research Laboratory, Department of Physics, Tezpur University, Napaam-784028, Assam, India

\begin{abstract}
Surface plasmon resonance SPR basically refers to collective oscillation of conduction electrons. Having the intrinsic property of sensing the surface properties, SPR have already received tremendous attention in the field of biomedical, Nano sensors, photovoltaics etc. Scientific data have also revealed that it is also highly characterized by the structure, type and dimension of the host nanoparticles, which eventually can lead us to a systematic correlation between different physical attributes of the host nanoparticles and SPR. In this work, we try to make a simplistic approach of correlating size, shape of gold $(\mathrm{Au})$ and silver $(\mathrm{Ag})$ nanoparticles (NPs) with their corresponding peak wavelength. It has also been attempted to bring the concentrations in the same frame. The computed results and the observed results are compared and matching up to a considerable extent has been shown in this work. It is envisioned that this small-scale correlation will help in giving a first insight towards characterization of Au and AgNPs just based only on SPR peak.
\end{abstract}

Keywords: SPR; Nanoparticles; MATLAB

\section{Introduction}

Every property of a material has a characteristic or critical length associated with it; e.g. scattering wavelength for electrical conductivity, plasma frequency (can be converted easily to wavelength) for optical property etc. The fundamental physics and chemistry changes when the dimensions of a solid become comparable to one or more of its characteristic wavelengths, many of which are in the nanometer range. Therefore, in the nano regime, novel physical and chemical properties can be experienced, e.g. yellow shiny bulk gold turn into red when the diameter of it is restricted to $10 \mathrm{~nm}$ [1-15]. One of the very tempting properties of the nanoparticles is that their properties depend on their size, shape and density. As for instance, in medical applications, the size of the nanoparticles should be less than $100 \mathrm{~nm}$. This is because, above this dimension, the nanoparticles become toxic to the living body. So, to implement nanoparticles in different applications, having reliable information about the size, shape and density is indeed imperative. In order to determine the size, shape and density we usually incorporate different techniques. TEM, SEM are used for determining the size and morphology, respectively. Similarly, AFM provides information of shape. These techniques are not in situ, some of them are costly and some processes are time consuming. Butwith the helpof some extraordinary properties of metal nanopar ticles called Surface Plasmon Resonance (SPR), the aspects likesize, shape and density of the metal nanoparticles can be feasibly extracted, saving of energy and time.

When total internal reflection occurs in a metal surface, then a wave moves along the surface of incidence which is referred to evanescent wave [2-5]. This wave occurs due to the interaction of electrons in the surface with the electric field of light. In case of metal bulk, the electrons absorb the light and oscillate exactly opposite to that of the incident wave and we perceive this as reflection of light. Therefore, metal appears to be shiny. But in case of metal nanoparticles, if the incident wave is nearly equal to that of the resonance frequency of the NPs, then they oscillate along with the electric field which is called Surface Plasmon Resonance. As such, they absorb a wavelength of light which we call SPR wavelength [1-7]. As the size of the nanoparticle changes, the resonance frequency of the particle changes. SPR wavelength also changes. The above descriptions eventually imply that there may be a quite sound correlation among the SPR wavelength, size, shape and density which can turn out to be standard equation [5-8].

Standardization of the equation can help the researchers to predict the size, shape and density of the sample without going into further investigations. So, this study is mainly aimed to find 
out a standard relation between the SPR wavelength and the physical aspects of nanoparticles. Due to dearth of abundant data, present study concentrates only to size and concentration of the nanoparticles.

\section{Data Sets and Methodology}

For formulating an empirical equation, data have been used which is listed in the Table 1 and customized code has been used in the MATLAB platform. In order to validate the equation, another data set as listed in the Table 2 has been used and standard deviations have been computed and plotted to see the accuracy of the formulated equation.

\section{Calculations for gold nanoparticles}

For the calculation of equation relating the concentration, size and wavelength, we had used the data already provided in literature listed in Table 1.

Table 1: Concentration, size and SPR peak wavelength of gold nanoparticle.

\begin{tabular}{|c|c|c|}
\hline Conc. (ppb)(cx109) & Size(nm) & 5 \\
\hline 5470 & 10 & 517 \\
\hline 598 & 15 & 519 \\
\hline 164 & 20 & 524 \\
\hline 65.4 & 50 & 526 \\
\hline 17.9 & 40 & 530 \\
\hline 7.15 & 50 & 535 \\
\hline 3.51 & 60 & 540 \\
\hline 1.96 & 500 & 5 \\
\hline
\end{tabular}
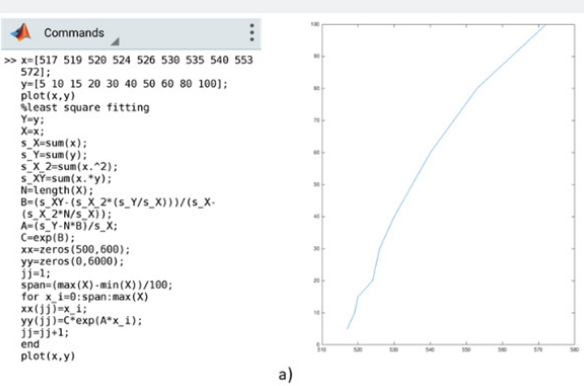

Figure 1A: MATLAB code for plotting size vs SPR wavelength graph and the plotted graph.

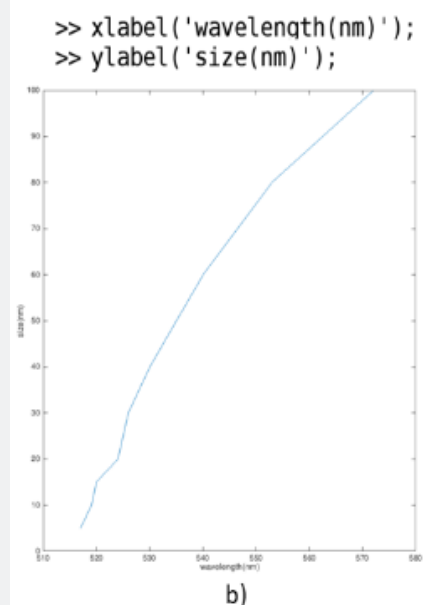

Figure 2: Code for labeling of the axes. 


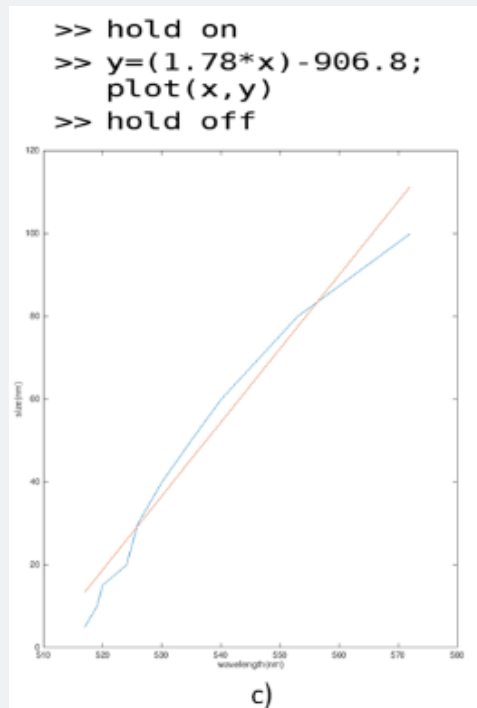

Figure 3: Code and plotting of least square fitting curve for gold metal nanoparticles.

\section{Relation between size and spr wavelength of the nanoparticles}

The customized code and respective plots that are used for formulating a relation between the size and peak wavelength of SPR of gold nanoparticles are shown in Figure 1-3.
Accordingly, relation between size and wavelength is linear, which can be defined by equation

$$
s_{g}=(1.8 x-907) \text { (approx.) }
$$

where, $s_{g}$ is the size of gold nanoparticle and $x_{g}$ is the SPR wavelength of gold nanoparticles (Figure 4).

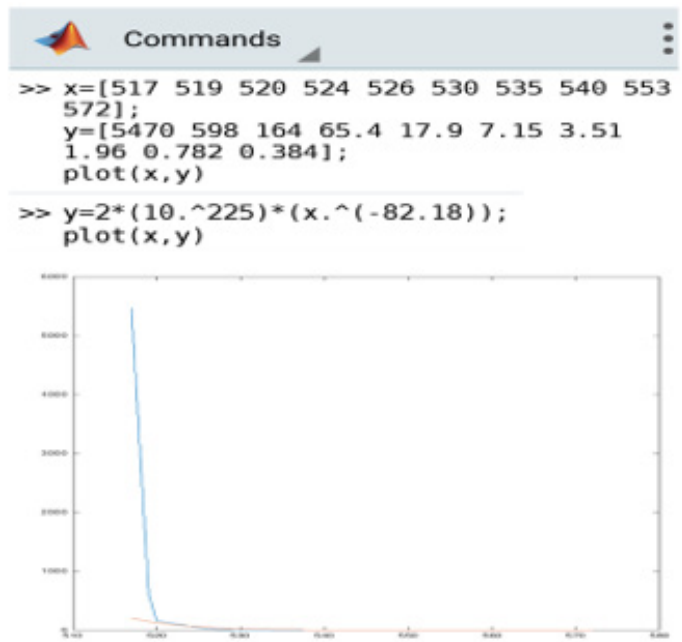

Figure 4: Code for plotting concentration vs wavelength graph and the equation of the curve for gold nanoparticles.

\section{Relation between concentration and wavelength}

Here, the equation provides relation between concentration and wavelength that is

$c_{g}=2 \times 10^{225} \times x_{g}-82.18$

In equation (2), $c_{g}$ refers to the concentration of the nanoparticles and $x_{g}$ is the SPR wavelength. Combining equations (1) and
(2), we derive the relation between SPR wavelength and size and concentration of the nanoparticles simultaneously, viz

$x_{g}=0.36 \times s_{g}+196 \times 10^{-0.012 \log c_{g}}+324$ (3)

where $x_{g}$ is the SPR wavelength, $s_{g}$ is the size and ${ }^{c} g$ is the concentration of the nanoparticles. Here $c_{g}$ is in $\mathrm{ppb}\left(\times 10^{19}\right)$ and $s_{g}$ is in $\mathrm{nm}$. The deviation of the calculated values from the experimental values of SPR is provided in Table 2 below. 
Table 2: Deviation of calculated from experimental values.

\begin{tabular}{|c|c|c|}
\hline SPR Wavelength (experimental) & SPR Wavelength (calculated) & Deviation \\
\hline 517 & 503 & 14 \\
\hline 519 & 509 & 10 \\
\hline 520 & 514 & 6 \\
\hline 524 & 518 & 6 \\
\hline 526 & 524 & 2 \\
\hline 530 & 530 & 0 \\
\hline 535 & 535 & 0 \\
\hline 540 & 540 & 0 \\
\hline 553 & 545 & 8 \\
\hline 572 & 554 & 18 \\
\hline
\end{tabular}

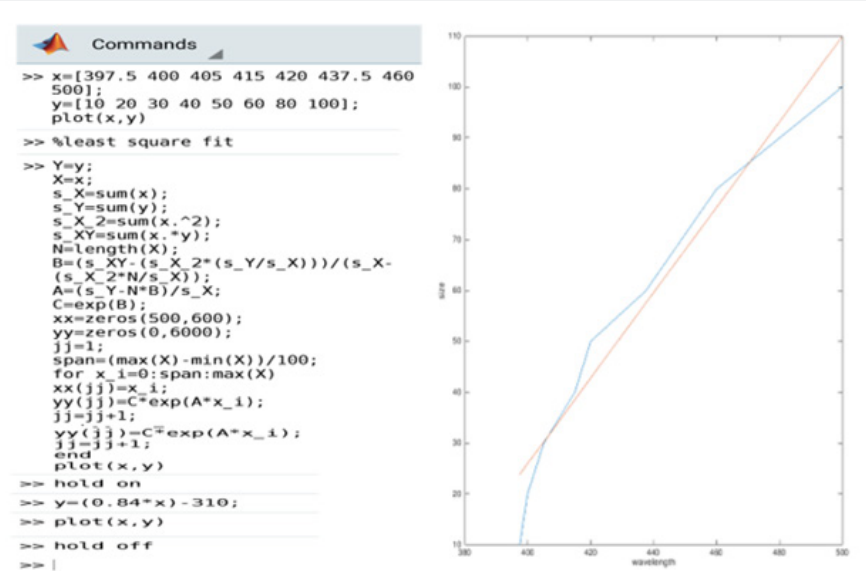

Figure 5: Customized code for plotting size vs. SPR wavelength graph and linear fitting of the graph and the equation of the linear fitting graph for silver nanoparticle.

Table 3: Deviation of calculated from experimental values.

\begin{tabular}{|c|c|c|}
\hline Conc.(ppb) (cx109) & Size(nm) & Peak Wavelength (SPR) \\
\hline 3600 & 10 & 497.5 \\
\hline 460 & 20 & 400 \\
\hline 140 & 30 & 415 \\
\hline 57 & 40 & 420 \\
\hline 29 & 50 & 437.5 \\
\hline 17 & 60 & 460 \\
\hline 7.1 & 80 & 500 \\
\hline 3.6 & 100 & \\
\hline
\end{tabular}

\section{Calculations for Silver Nanoparticles}

In similitude to AuNPs, we have performed for silver nanoparticles to establish correlation corresponding to data as listed in Table 3 (Figure 5).

\section{Relation Between Size and Wavelength}

Accordingly, we develop the relation between size and wavelength is

$s_{S}=\left(0.84 x_{S}-310\right)$ (approx) (4)

Where, $s_{s}$ is the size of silver nanoparticle and $x_{s}$ is the SPR wavelength (Figure 6)

\section{Relation between concentration and wavelength}

Likewise, the relation between concentration and wavelength is given by 
$c_{s}=2.64 \times 10^{66} \times x_{s}^{-24.65}(5)$

Combining both equations and, we get $x_{s}=0.54 \times s_{S}+266 \times 10^{-0.04 \log c_{S}}{ }_{-} 168.5$ (6)

where ${ }^{c}$ is in $p p b\left(\times 10^{18}\right)$ and $s_{s}$ is in $\mathrm{nm}$.

\section{Deviation of the theoretical data from experimental data}

Deviation between theoretical and calculated values is listed in Table 4.
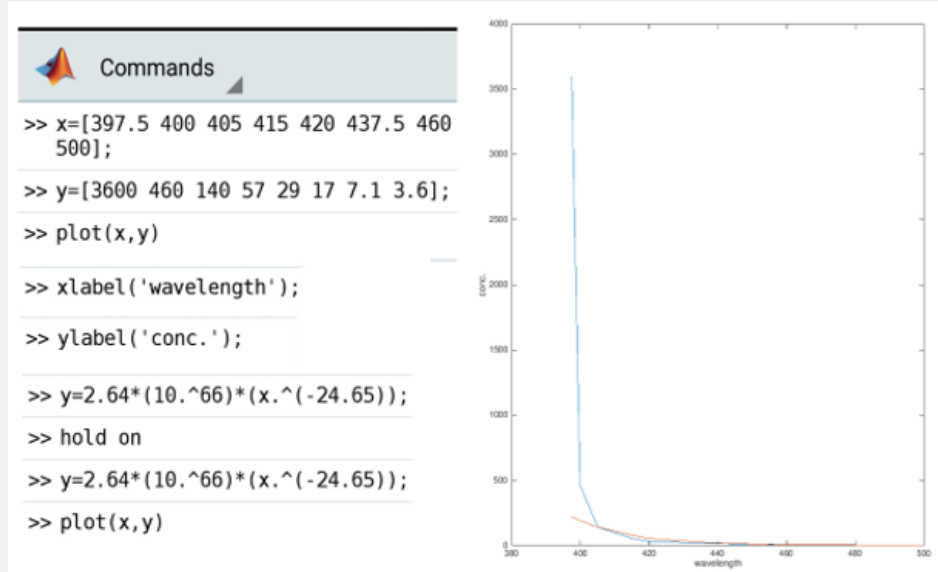

Figure 6: Customized code for plotting concentration vs. SPR wavelength graph and linear fitting of the graph and the equation of the linear fitting graph for silver nanoparticle.

Table 4: Comparison of experimental and computed values..

\begin{tabular}{|c|c|c|}
\hline Peak Wavelength (SPR) & Wavelength (calculated) & Deviation \\
\hline 397.5 & 366 & 31.5 \\
\hline 400 & 387 & 13 \\
\hline 405 & 403 & -1 \\
\hline 415 & 416 & -8 \\
\hline 420 & 428 & -0.5 \\
\hline 437.5 & 438 & 2 \\
\hline 460 & 458 & 25 \\
\hline 500 & 475 & 2 \\
\hline
\end{tabular}

\section{Results and Discussions}

In summary, we have tried to formulate correlation between parameters of nanoparticles affecting surface Plasmon resonance wavelength of gold and silver nanoparticles. The available data lead us to two different equations, one for silver and one for gold nanoparticles which correlate their size, concentration and wavelength. For gold nanoparticles, the equation came out to be $x_{g}=0.36 \times s_{g}+196 \times 10^{-0.012 \log c_{g}}+324$

Where conc. is in $p p b\left(\times 10^{18}\right)$ and size is in nm. For silver nanoparticles, the equation came out to be

$$
x_{S}=0.54 \times s_{S}+266 \times 10^{-0.04 \log c_{S}}{ }_{-} 168.5
$$

Where conc. is in $p p b\left(\times 10^{18}\right)$ and size is in nm. These equations are then verified with respect to experimental dataset. Our observations reveal that there is matching of the values to a con- siderable extent. There is lot of scope for development of this work. This work will give us a priori about which concentration of the given solution of nanoparticle of known size will give wavelength (SPR) in required range. The empirical formulations will provide a quicker way to characterize the $\mathrm{Au}$ and AgNPs through the allied parameters..

\section{References}

1. Ahmad T, Wani IA, Ahmed J, Al-Hartomy OA (2014) Effect of gold ion concentration on size and properties of gold nanoparticles in TritonX-100 based inverse microemulsions. Applied Nanoscience 4(4): 491-498.

2. Rahman S (2016) Size and concentration analysis of gold nanoparticles with ultraviolet-visible spectroscopy. Undergraduate Journal of Mathematical Modeling: One+ Two 7(1): 1-15.

3. Link S, El-Sayed MA (1999) Size and temperature dependence of the plasmon absorption of colloidal gold nanoparticles. The Journal of Physical Chemistry B 103(21): 4212-4217. 
4. Shameli K, Ahmad MB, Jazayeri SD, Sedaghat S, Shabanzadeh P, et al. (2012) Synthesis and characterization of polyethylene glycol mediated silver nanoparticles by the green method. Int J Mol Sci 13(6): 66396650 .

5. Paul D, Dutta S, Biswas R (2016) LSPR enhanced gasoline sensor. Journal of Physics D: Applied Physics 49(30): 305104.

6. Paul D, Dutta S, Saha D, Biswas R (2017) LSPR based Ultra-sensitive low-cost U-bent optical fiber for volatile liquid sensing. Sensor and Actuators B (Chemical) 250: 198-207.

7. Baruah BS, Biswas R (2018) Selective detection of arsenic (III) based on colorimetric approach in aqueous medium using functionalized gold nanoparticles unit. Material Research Express 5(1).

8. Paul D, Biswas R (2018) Multiple material domains based moda parameter analysis of photonic crystal fiber. Laser Research and Applications Journal 1: 002

9. Paul D, Biswas R (2018) Facile fabrication of sensing set-up for size detection of nanoparticles. IEEE Transactions on Nanotechnology 17(3): 596-602.

10. Baruah BS, Biswas R (2018) Localized surface plasmon resonancebased U-shaped optical fiber probe for the detection of $\mathrm{Pb}^{2+}$ in aqueous medium. Sensors and Actuators B Chemical 276: 89-94.
11. B Baruah BS, Biswas R (2018) An optical fiber-based surface plasmon resonance technique for sensing of lead ions: A toxic water pollutant. Optical Fiber Technology 46: 152-156.

12. Baruah BS, Biswas R, Deb P (2019) A green colorimetric approach towards detection of arsenic (III): a pervasive environmental pollutant. Optics and Laser Technology 111: 825-829.

13. Baruah BS, Biswas R (2019) Mangifera indica leaf extract mediated gold nanoparticles: a novel platform for sensing of As(III). IEEE Sensors Letter 3(3).

14. Boruah BS, Daimari NK, Biswas R (2019) Functionalized silver nanoparticles as an effective medium towards trace determination of arsenic (III) in aqueous solution, Results in Physics 12: 2061-2065.

15. Boruah BS, Biswas R (2019) Probing lead ion contamination in aqueous solution through bio-inspired surface modification of gold nanoparticles on D-shaped fiber. IEEE Transactions on Nanotechnology 18: 770-775. 